\title{
BAG Family Molecular Chaperone Regulator 5
}

National Cancer Institute

\section{Source}

National Cancer Institute. BAG Family Molecular Chaperone Regulator 5. NCI Thesaurus.

Code C26144.

BAG family molecular chaperone regulator 5 ( $447 \mathrm{aa}, \sim 51 \mathrm{kDa}$ ) is encoded by the human BAG5 gene. This protein is involved in the negative regulation of apoptosis, the inhibition of protein ubiquitination and the modulation of protein folding. 